\title{
ФОРМИРОВАНИЕ НАВЫКОВ САМООБСЛУЖИВАНИЯ В ПРОГРАММЕ ВОССТАНОВИТЕЛЬНОГО ОБУЧЕНИЯ ПАЦИЕНТОВ МОЛОДОГО ВОЗРАСТА С ОСТРЫМИ НАРУШЕНИЯМИ МОЗГОВОГО КРОВООБРАЩЕНИЯ
}

\author{
(С) Никишина В.Б. ${ }^{l}$, Петраш Е.А. ${ }^{l}$, Шутеева Т.В. ${ }^{l}$, Стороженко М.В. ${ }^{2}$, Савостиков В.А. ${ }^{l}$ \\ ${ }^{1}$ Курский государственный медицинский университет, Курск; \\ ${ }^{2}$ Курский областной санаторий «Соловьиные зори», Курск \\ E-mail: petrash@mail.ru
}

\begin{abstract}
В статье представлены результаты исследования по формированию навыков самообслуживания в программе восстановительного обучения постинсультных пациентов молодого возраста. Сравнительный анализ функций праксиса у постинсультных пациентов молодого возраста до и после восстановительного обучения позволил выявить значимое повышение темпа и точности кинетического, кинестетического и пространственно-динамического праксиса; повышение дифференцированности и координированности серийной организации движений и действий. У постинсультных пациентов молодого возраста с поражением не ведущего (правого) полушария головного мозга отмечается более интенсивная динамика как по восстановлению функций праксиса (оцениваемых по критериям темпа, точности, дифференцированности и координированности), так и по формированию мелкомоторных навыков самообслуживания в сравнении с пациентами с локализацией очага поражения в ведущем (левом) полушарии. Интенсивность восстановления функции с последующим формированием навыка обусловлена включенностью не ведущего полушария и взаимосвязана с повышением точности и темпа моторных функций.
\end{abstract}

Ключевые слова: навыки самообслуживания, восстановительное обучение, пациенты молодого возраста с острыми нарушениями мозгового кровообращения.

\section{FORMATION OF SELF-SERVICE SKILLS IN THE PROGRAM OF RETROACTIVE TRAINING OF YOUNG PATIENTS WITH ACUTE DISORDERS OF CEREBRAL CIRCULATION Nikishina V.B. ${ }^{1}$, Petrash E.A. ${ }^{l}$, Shuteeva T.V. ${ }^{1}$, Storozhenko M.V. ${ }^{2}$, Savostikov V.A. ${ }^{l}$ \\ ${ }^{1}$ Kursk State Medical University, Kursk; \\ ${ }^{2}$ Kursk Regional Sanatorium "Solov'inye Zori" ("Nightingale Dawns"), Kursk}

The article presents the results of investigating the self-service skills formation in the program of restorative education for young post-stroke patients. Comparative analysis of the praxis functions in post-stroke young patients before and after rehabilitation training made it possible to reveal a significant increase in the rate and accuracy of the kinetic, kinesthetic, and spatiotemporal praxis; increasing the differentiation and coordination of the seriated organization of movements and actions. In poststroke young patients with a lesion of the non-leading (right) cerebral hemisphere, a more intensive dynamics is observed both in restoring the praxis functions (estimated according to the criteria of pace, accuracy, differentiation and coordination), and in developing small self-service skills in comparison with patients with the lesion in the dominant (left) hemisphere. The intensity of functional restoration with the subsequent formation of the skill is due to the involvement of the non-leading hemisphere and is interrelated with an increase in the accuracy and pace of motor functions.

Keywords: self-service skills, rehabilitation education, young patients with acute disorders of cerebral circulation.

Согласно данным статистико-демографических отчетов Минздрава РФ, количество смертельных исходов за последние 5 лет (20122017 г.г.) от цереброваскулярных болезней сокращается [3]. Тогда как по распространенности заболеваемости инфарктом мозга отмечается тенденция положительной ее динамики. То есть количество пациентов, попадающих в зону активной реабилитации, с данной нозологией, очевидно, растет. В ряде исследовательских и статистических обзоров также отмечается существенное снижение возрастных границ возникновения острых нарушений мозгового кровообращения (ОНМК) до 35-40 лет [7]. Молодым возрастом для возникновения ОНМК является хронологический возраст до 45 лет. В связи с этим актуальной является необходимость реабилитации и восстано- вительного обучения постинсультных пациентов молодого возраста, перенесших ОНМК. Существенная часть функциональных нарушений при данной нозологии связана с моторнодвигательной сферой (как мелкомоторной, так и грубомоторной) на всех уровнях ее организации: рефлекторном, локомоторном, произвольном, автоматизированном.

В настоящее время программы реабилитации и восстановительного обучения ориентированы преимущественно на восстановление грубомоторных функций (ходьба, поддержание позы тела и др.) и локомоций верхних конечностей $[9,10]$. Восстановительному обучению, направленному на поддержание мелкомоторных действий, не уделяется достаточно внимания. Именно произвольные мелкомоторные действия составляют 
основу для формирования автоматизированных действий [1], которые складываются в навыки самообслуживания постинсультных пациентов.

Методологическое построение программы восстановительного обучения постинсультных пациентов молодого возраста осуществлялось на принципах нейроэволюционной теории Дж. Эдельмана: нейроны в головном мозге когнитивно или поведенчески специализированы; «используй или потеряешь» (функционирующие нейроны борются за место связывания; механизм конкуренции лежит в основе формирования функций); «работающие вместе, связываются вместе» (направление связывания нейронов от сенсорной и моторной коры к ассоциативной; депривация нейронов как их нефункционирование приводит к необратимым последствиям на уровне сенсорной и моторной коры) [8].

Моторно-двигательные функции имеют иерархическую структуру, включающую рефлекторный, локомоторный, произвольный и автоматизированный уровни организации движений. Морфофункциональная организация движений на рефлекторном уровне обеспечивается активностью спинного мозга и стволовых отделов головного мозга; на локомоторном уровне включает в себя субталамус, спинной мозг, красное ядро среднего мозга, ретикулярные ядра моста, вестибулярные ядра продолговатого мозга и моста, мозжечок и базальные ганглии. На произвольном уровне морфофункциональная организация движений обеспечивается прецентральной извилиной лобной коры головного мозга, моторной корой (4,6 поля по Бродману), корой поясной извилины и ассоциативной лобной корой; автоматизированный уровень движений обеспечивается базальными ганглиями, мозжечком и ассоциативной лобной корой. Представленные уровни организации движений реализуются в трех функциональных блоках мозга (по А.Р. Лурия): энергетическом блоке, блоке приема, переработки и хранения информации и блоке программирования, регуляции и контроля деятельности [4]. Нарушения рефлекторного уровня организации движений функционально соответствуют первому блоку мозга - энергетическому; проявления локомоторных нарушений соответствуют второму функциональному блоку мозга; нарушения произвольных и автоматизированных движений функционально соответствуют третьему блоку мозга $[5,6$, $11]$.

Целью исследования является изучение эффективности формирования навыков мелкомоторного самообслуживания в программе восстановительного обучения пациентов молодого возраста с острыми нарушениями мозгового кровообращения.

\section{МАТЕРИАЛЫ И МЕТОДЫ ИССЛЕДОВАНИЯ}

Общий объем исследовательской выборки составил 19 человек (11 мужчин и 8 женщин) в возрасте 27-44 года $(32,48 \pm 4,29$ года), впервые перенесших острое нарушение мозгового кровообращения. Все пациенты были госпитализированы в течение первых суток после перенесенного инфаркта мозга. Исследование проводилось в раннем восстановительном периоде; все пациенты находились на реабилитации в неврологическом отделении санатория. Основанием включения испытуемых в исследовательские группы являлись: полушарная локализация очага ишемического инсульта в теменно-височной области коры головного мозга; левополушарный профиль латерализации (по результатам оценки профиля латерализации с использованием «Методики автоматизированного исследования индивидуального латерального профиля» (студия ВиЭль) все пациенты являлись праворукими). В первую группу испытуемых вошли 8 человек с локализацией очага поражения в левом (ведущем) полушарии головного мозга; вторую группу составили 11 пациентов молодого возраста, перенесших ишемический инсульт, с правополушарной локализацией очага поражения (в не ведущем полушарии). У 5 пациентов был диагностирован кардиоэмболический ишемический инсульт, у 5 - атеротромботический, криптогенный ишемический инсульт без признаков системной гиперкоагуляции был диагностирован у 7 пациентов, с признаками коагулопатии неуточненного генеза - у 2.

Для подтверждения локализации очага и характера инсульта использовались методы нейровизуализации: компьютерная томография (КТ) и(или) магнито-резонансная томография (МРТ). В неврологическом статусе были выявлены гемипарезы умеренной степени выраженности той или иной латерализации, центральные парезы 7 и 12 пар черепно-мозговых нервов, расстройства различных видов чувствительности (по гемитипу). По результатам когнитивного тестирования в данной группе пациентов с использованием шкалы MMSE были выявлены легкие когнитивные расстройства, одновременно проявляющиеся в нескольких когнитивных сферах.

Организация основного исследования осуществлялась последовательно в два этапа: диагностический этап и этап восстановительного обучения. Задачи и методы диагностического этапа представлены в таблице 1. 
Задачи и методы диагностического этапа исследования

\begin{tabular}{|c|c|}
\hline Задача & Метод \\
\hline $\begin{array}{l}\text { Оценка гностических } \\
\text { функций (пространственного } \\
\text { и символического гнозиса) }\end{array}$ & $\begin{array}{l}\text { Функциональные нейропсихологические пробы: «Слепые часы»; «Бочка и } \\
\text { ящик»; проба на узнавание символьных изображений (цифр и букв). }\end{array}$ \\
\hline $\begin{array}{c}\text { Оценка моторных функций по } \\
\text { уровням: }\end{array}$ & \\
\hline - рефлекторный уровень; & $\begin{array}{l}\text { Оценка карпорадиального рефлекса верхних конечностей; сухожильных } \\
\text { рефлексов с двуглавой и трехглавой мышц верхних конечностей. }\end{array}$ \\
\hline - локомоторный уровень; & $\begin{array}{l}\text { Оценка поддержания позы тела (сидя); ориентация собственного тела во } \\
\text { внешнем пространстве (верх-низ, право-лево). }\end{array}$ \\
\hline - произвольный уровень; & $\begin{array}{l}\text { Функциональные нейропсихологические пробы, направленные на оценку } \\
\text { кинетического, кинестетического, пространственно-динамического } \\
\text { праксиса. }\end{array}$ \\
\hline $\begin{array}{l}\text { - автоматизированный } \\
\text { уровень. }\end{array}$ & $\begin{array}{l}\text { Выполнение функциональных символических действий, соответствующих } \\
\text { навыкам самообслуживания: навыки организации собственного питания } \\
\text { (самостоятельная еда ложкой); навыки одевания/раздевания } \\
\text { (застегивание/расстегивание молнии); навыки безопасности } \\
\text { (самостоятельный набор телефонного номера). }\end{array}$ \\
\hline
\end{tabular}

Оценка результатов выполнения функциональных нейропсихологических проб, направленных на исследование функций праксиса и автоматизированных действий (по критериям темпа, точности, координированности), а также пространственного и символического гнозиса, осуществлялась по 4-балльной шкале (Вассерман Л.И., 1997): 0 баллов - отсутствие ошибок или «неспецифические» ошибки для той или иной пробы, свойственные и здоровым испытуемым; 1 балл - слабовыраженные нарушения, отмечается ряд мелких погрешностей, исправляемых самостоятельно (нижняя нормативная граница); 2 балла - нарушения высших психических функций средней степени тяжести (испытуемый в состоянии выполнить задание после нескольких попыток, развернутых подсказок и наводящих вопросов); 3 балла - грубые расстройства высших психических функций (задание недоступно для выполнения даже после подробного многократного разъяснения со стороны экспериментатора) [2].
Программа восстановительного обучения постинсультных пациентов включала в себя три этапа, которые формировались в соответствии с уровнями организации движений: локомоторный уровень, уровень произвольной регуляции, уровень автоматизированных движений.

На локомоторном уровне все движения осуществлялись сначала с помощью, затем по зрительному образцу, потом самостоятельно в следующей последовательности: 1) ритмическое сгибание/разгибание лучезапястного и локтевого суставов с увеличением ритма (грубомоторные движения); 2) движения руками с имитацией шага в нескольких вариантах (левая нога - левая рука; правая нога - правая рука; левая нога - правая рука; правая нога - левая рука); 3) ритмическое сгибание/разгибание пальцев рук (отдельно левой рукой, правой рукой, двумя руками одновременно). На произвольном уровне выполнялась следующая последовательность задач восстановительного обучения: 1) серийная организация движений со зрительно-сенсорным контролем; со слухо-сенсорным контролем; 2) дивергенция 
символического навыка самообслуживания (разложить действие на отдельные двигательные акты вербально; разложить действие на отдельные символические движения; разложить действие на отдельные моторные акты); 3) воспроизведение символического действия в обратном порядке; 4) конвергенция символических действий по перемещению объекта в горизонтальной плоскости (вертикально, горизонтально, по диагонали); 5) конвергенция символических действий по перемещению объекта в трехмерном пространстве. На уровне автоматизации последовательность задач восстановительного обучения имела следующий вид: 1) автоматизация кинетического, кинестетического, пространственно-динамического компонентов серийной организации движений; 2) автоматизация серии мелкомоторных движений, составляющих навыки самообслуживания (самостоятельная еда ложкой; застегивание/расстегивание молнии; самостоятельный набор телефонного номера).

Каждое занятие программы восстановительного обучения предполагает последовательную активацию локомоторного, произвольного и уровня автоматизации движений. Алгоритм построения занятия по восстановительному обучению включает в себя выполнение обучающих заданий в определенной последовательности и с учетом изменения доли временного интервала, отведенного для активации каждого уровня организации движения (локомоторного, произвольного, автоматизированного) в общей продолжительности занятия. Хронологическая длительность восстановительного обучения по формированию автоматизированных мелкомоторных движений (навыков самообслуживания) постинсультных пациентов составила 20 дней с ежедневной периодичностью занятий (по два занятия в день - в первой и второй половине дня). Продолжительность занятий в процессе восстановительного обучения варьировалась - от 15 до 35 минут, постепенно увеличиваясь к окончанию курса восстановительного обучения.

Статистическая обработка полученных результатов осуществлялась с использованием методов описательной (показатели средних значений, среднее квадратическое отклонение) и сравнительной статистики (непараметрического критерия $\chi^{2}-$ Фридмана, $\left.\mathrm{p}<0,05\right)$.

\section{РЕЗУЛЬТАТЫ ИССЛЕДОВАНИЯ И ИХ ОБСУЖДЕНИЕ}

В результате оценки исходного уровня гностических функций у постинсультных пациентов молодого возраста (до восстановительного обучения) вне зависимости от полушарной локализа- ции очага ишемического инсульта выявлено нарушение символического и пространственного гнозиса. Нарушение пространственного гнозиса проявляется в снижении точности право-левой ориентировки во внешнем пространстве при сохранности ориентировки в квазипространстве (в собственном теле). Нарушение символического гнозиса (узнавание цифровых и буквенных обозначений) проявляется в том, что пациенты не фиксируют ошибки пространственного расположения букв и цифр.

В результате предварительной оценки моторных функций у постинсультных пациентов молодого возраста до восстановительного обучения на рефлекторном уровне все пациенты характеризуются отсутствием спинальных нарушений. На локомоторном уровне вне зависимости от полушарной локализации очага поражения у всех групп пациентов выявлено снижение точности право-левой ориентировки во внешнем пространстве при сохранности ориентации в собственном теле и удержании позы тела в течение 30-35 минут. На произвольном уровне выявлено снижение темпа и точности кинетического, кинестетического, пространственно-динамического праксиса. Нарушения произвольных движений и действий проявляются в затруднениях воспроизведения поз пальцев кистей рук как по зрительному, так и по кинестетическому образцу. Постинсультные пациенты молодого возраста с локализацией очага поражения в ведущем (левом) полушарии при воспроизведении необходимого положения на руке, контрлатеральной очагу поражения, помогают себе второй рукой. Пациенты с локализацией очага поражения в не ведущем полушарии (правом) находят нужную позу методом подбора. Проявлением нарушения пространственнодинамического праксиса являются ошибки в право-левой ориентировке, а также снижение реципрокной координации движений. У постинсультных пациентов молодого возраста отмечаются два типа проявлений снижения реципрокной координации движений: синхронное выполнение действий правой и левой рукой; отставание темпа действий руки, контрлатеральной очагу поражения. При выполнении заданной последовательности действий («кулак-ребро-ладонь»), выявлено нарушение выполнения моторной программы, что проявляется в ее упрощении (пропуск одного из звеньев программы); либо персевераторном повторении одного и того же элемента такое количество раз, сколько звеньев содержит исходный образец моторной программы; либо внесении дополнительных элементов программы.

При оценке автоматизированного уровня организации движений у постинсультных пациентов молодого возраста вне зависимости от полу- 
шарной локализации очага поражения до восстановительного обучения выявлена невозможность выполнения символических действий, соответствующих навыкам самообслуживания (самостоятельная еда, застегивание/расстегивание молнии, набор телефонного номера), что обусловлено невозможностью самостоятельного формирования и фиксации моторной программы. Выполнение отдельных символических движений и действий, составляющих мелкомоторные навыки самообслуживания, постинсультным пациентам вне зависимости от полушарной локализации очага поражения является доступным.

После завершения курса восстановительного обучения постинсультных пациентов молодого возраста была проведена повторная оценка моторных функций в соответствии с выделенными уровнями организации движений (локомоторным, произвольным, автоматизированным) по группам испытуемых. В результате была выявлена положительная динамика по всем исследуемым показателям (таблица 2).

В результате исследования достоверно установлено, что у пациентов молодого возраста, перенесших ОНМК (ишемический инсульт), в уровневой структуре организации движений преобладающим является уровень произвольных движений и действий при равнозначной представленности рефлекторного и автоматизированного уровней, в то время как в норме движения, соответствующие произвольному и автоматизированному уровням, совокупно составляют $2 / 3$ от общего объема движений.

Сравнительный анализ функций праксиса у постинсультных пациентов молодого возраста позволил выявить значимое повышение темпа и точности кинетического, кинестетического и пространственно-динамического праксиса. Выявлено значимое повышение дифференцированности и координированности серийной организации движений и действий. Учитывая обратный характер оценочной шкалы, снижение количественного показателя свидетельствует о значимой положительной динамике (рис. 1).

Таким образом, в результате исследования у постинсультных пациентов молодого возраста с поражением не ведущего (правого) полушария головного отмечается более интенсивная динамика как по восстановлению функций праксиса (оцениваемых по критериям темпа, точности, дифференцированности и координированности), так и по формированию мелкомоторных навыков самообслуживания в сравнении с пациентами с локализацией очага поражения в ведущем (левом) полушарии. Интенсивность восстановления функции с последующим формированием навыка обусловлена включенностью не ведущего полушария и взаимосвязана с повышением точности и темпа моторных функций.

Таблица 2

Показатели значимости различий показателей двигательных функций у постинсультных пациентов молодого возраста до и после восстановительного обучения ( $\chi^{2}$ Фридмана, $\left.\mathrm{p}<0,05\right)$

\begin{tabular}{|c|c|c|c|}
\hline & Показатели & $\begin{array}{c}\text { Поражение } \\
\text { ведущего (левого) } \\
\text { полушария } \\
\end{array}$ & $\begin{array}{c}\text { Поражение не } \\
\text { ведущего (правого) } \\
\text { полушария } \\
\end{array}$ \\
\hline \multirow{3}{*}{$\begin{array}{c}\text { Произвольный } \\
\text { уровень движений }\end{array}$} & Кинетический праксис & $0,029^{*}$ & $0,034^{*}$ \\
\hline & Кинестетический праксис & $0,038^{*}$ & $0,018^{*}$ \\
\hline & $\begin{array}{l}\text { Пространственно-динамический } \\
\text { праксис }\end{array}$ & $0,019^{*}$ & $0,029 *$ \\
\hline \multirow{3}{*}{$\begin{array}{c}\text { Автоматизированный } \\
\text { уровень движений }\end{array}$} & $\begin{array}{l}\text { Навык самостоятельного приема } \\
\text { пищи }\end{array}$ & $0,007 *$ & $0,051^{*}$ \\
\hline & $\begin{array}{l}\text { Навык самостоятельного } \\
\text { застегивания/расстегивания молнии }\end{array}$ & $0,012 *$ & $0,044^{*}$ \\
\hline & $\begin{array}{l}\text { Навык самостоятельного набора } \\
\text { телефонного номера }\end{array}$ & 0,034 & $0,026^{*}$ \\
\hline
\end{tabular}

Примечание: * - статистическая значимость различий показателей двигательных функций до и после восстановительного обучения у пациентов с поражением ведущего (не ведущего) полушария. 
Уровни организации движений

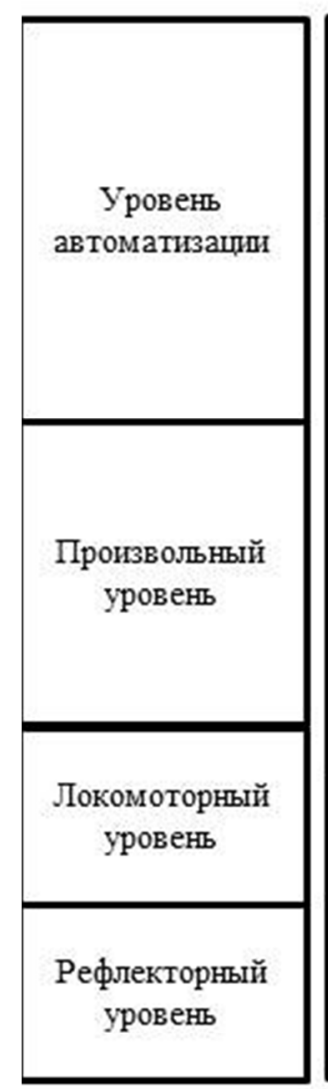

HOPMA

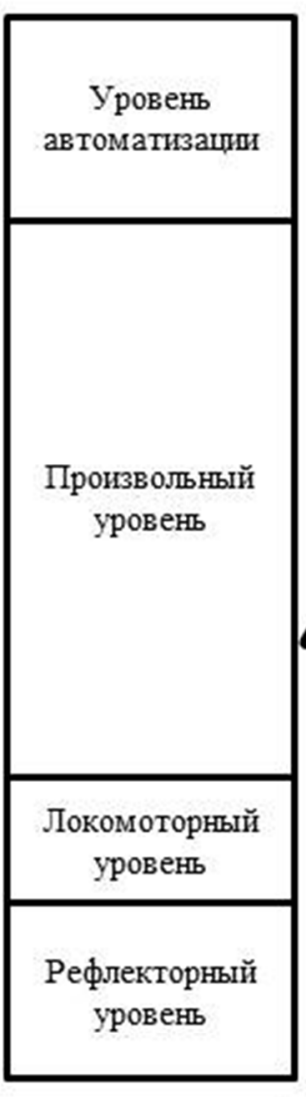

ИШЕМИЧЕСКИЙ ИнсуЛЬТ

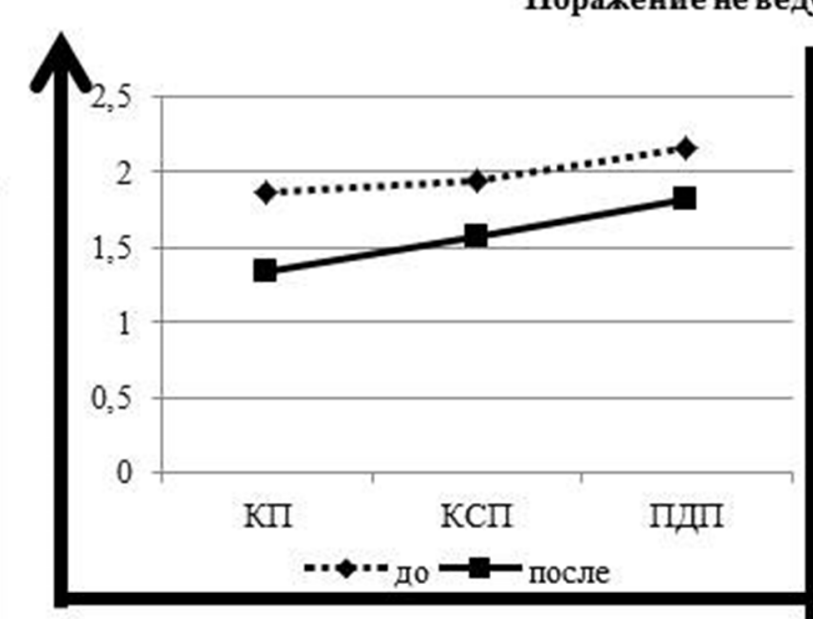

Произвольный уровень организации двикений

Поражение ведущего потушария

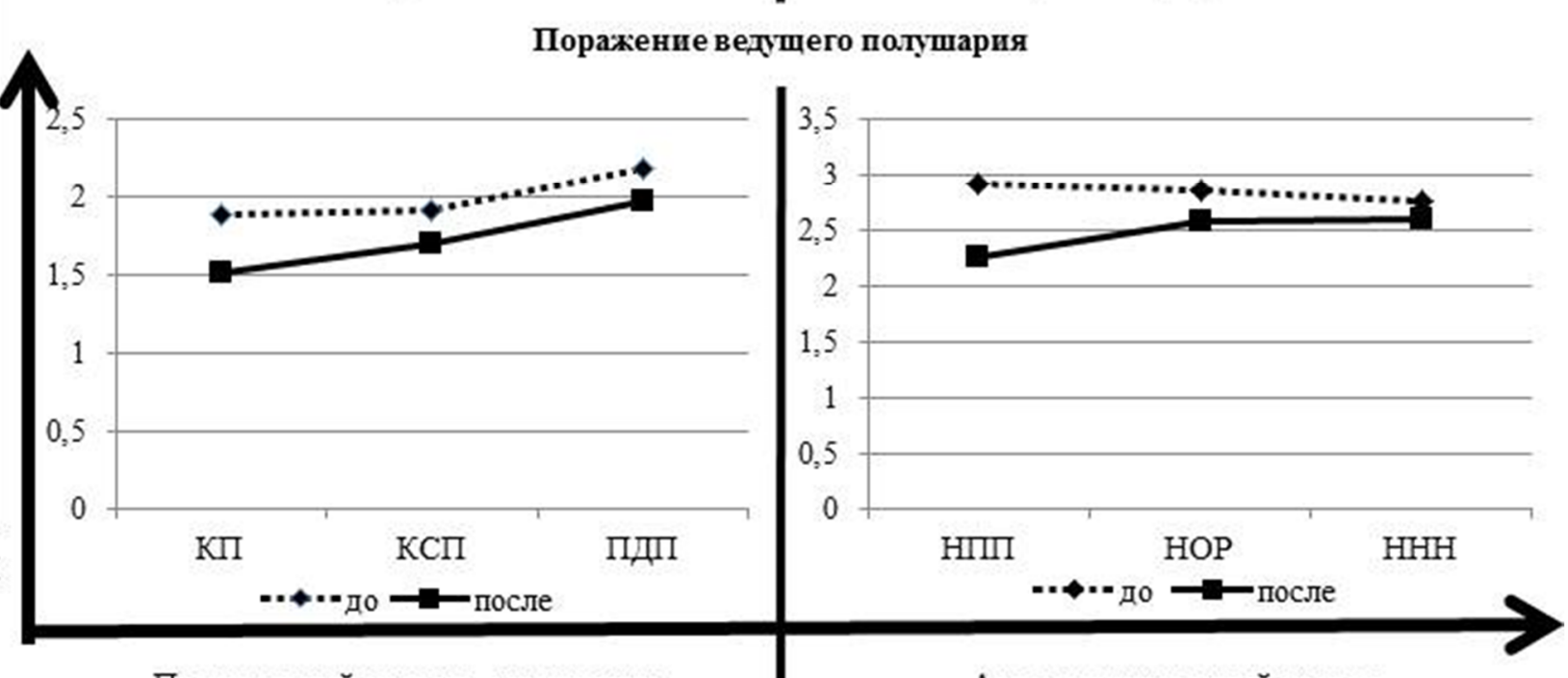

Произвольный уровень организации двикений

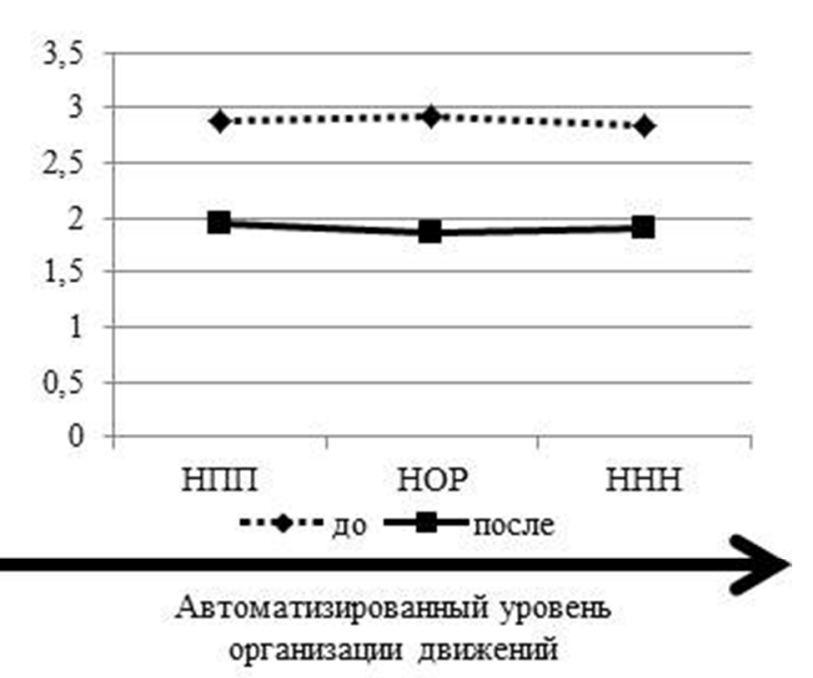

Автоматизированный уровень организации двикений

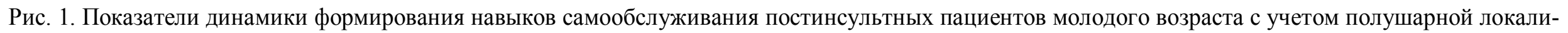
зации очага поражения.

Условные обозначения: КП - кинетический праксис, КСП - кинестетический праксис; ПДП - пространственно-динамический праксис; НПП - навык самостоятельного приема пищи; НОР - навык самостоятельного одевания/раздевания; ННН - навык самостоятельного набора телефонного номера. 


\section{ЛИТЕРАТУРА / REFERENCES}

1. Александров Ю.И. Психофизиологические закономерности научения и методы обучения // Психологический журнал. - 2012. - Т. 33, № 6. - С. 5-19. [Alexandrov Yu.I. Psychophysiological regularities of learning and methods of training. Psikhologicheskiy zhurnal. 2012; 1(6): 5-19 (in Russ.)].

2. Вассерман Л.И., Дорофеева С.А., Меерсон Я.А. Методы нейропсихологической диагностики: Практическое руководство. - СПб. : Стройлеспечать, 1997. - 400 c. [Vasserman L.I., Dorofeeva S.A., Meerson Ya.A. Neuropsychological diagnostic methods: a practical guide. SPb. : Stroylespechat'; 1997: 400 (in Russ.)].

3. Демографический ежегодник России. 2017: Стат. сб. - М. : Росстат, 2017. - 263 с. [Demographic Yearbook of Russia. 2017. M. : Rosstat; 2017: 263 (in Russ.)].

4. Лурия А.P. Высшие корковые функции человека. СПб. : Питер, 2008. - 624 с. [Luriya A.R. Higher cortical functions of man. SPb. : Piter; 2008: 624 (in Russ.)].

5. Никишина В.Б., Петраш Е.А., Запесоикая И.В. Восприятие эмоций у больных с ишемическим инсультом // Журнал неврологии и психиатрии им. С.С. Корсакова. - 2015. - Т. 115, № 10-1. - С. 4-9. [Nikishina V.B., Petrash E.A., Zapesotskaya I.V. Perception of emotions in patients with ischemic stroke. Zhurnal nevrologii i psikhiatrii im. S.S. Korsakova. 2015; 115(10-1): 4-9 (in Russ.)].

6. Никишина В.Б., Петраш Е.А. Реципрокная организация конструктивной деятельности у пациентов с ишемическим инсультом // Журнал неврологии и психиатрии им. С.С. Корсакова. - 2017. - Т. 117. -
№ 3-2. - C. 79-89. [Nikishina V.B., Petrash E.A. Reciprocal organization of constructive activity in patients with ischemic stroke. Zhurnal nevrologii $\mathrm{i}$ psikhiatrii im. S.S. Korsakova. 2017; 117(3-2): 79-89 (in Russ.)].

7. Ревегук Е.А., Карпов С.М. Распространенность ОНМК среди лиц молодого возраста // Успехи современного естествознания. - 2012. - № 5. C. 61-62; URL: http://naturalsciences.ru/ru/article/view?id=30084 (дата обращения: 11.04.2018). [Reveguk E.A., Karpov S.M. The prevalence of stroke among young adults. Uspekhi sovremennogo estestvoznaniya. 2012; (5): 61-2; URL: $\mathrm{http}: / /$ natural-sciences.ru/ru/article/view?id=30084 (in Russ.)].

8. Edelman G. Second Nature: Brain Science and Human Knowledge. - Yale : Yale University Press, 2006. $203 \mathrm{p}$.

9. Hawkins K.A., Fox E.J., Daly J.J., Rose D.K., Christou E.A., McGuirk T.E., Otzel D.M., Butera K.A., Chatterjee S.A., Clark D.J. Prefrontal over-activation during walking in people with mobility deficits: Interpretation and functional implications // Hum Mov Sci. - 2018. - Vol. 59. - P. 46-55. - DOI: 10.1016/j.humov.2018.03.010.

10. Israely S., Leisman G., Machluf C.C., Carmeli E. Muscle Synergies Control during Hand-Reaching Tasks in Multiple Directions Post stroke // Front Comput Neurosci. - 2018. - Vol. 12. - P. 10. - DOI: 10.3389/fncom.2018.00010

11. Nikishina V.B., Petrash E.A., Bushueva O.Y., Zapesotskaya I.V. Analysis of measures of functional asymmetry of the brain in patients with ischemic stroke: a neuropsychological study // Neuroscience and Behavioral Physiology. - 2016. - Vol. 46, N 4. - P. 408-412. 